\title{
Estilo de vida de adolescentes estudantes de escolas públicas e privadas em Recife: ERICA
}

\author{
Lifestyle of adolescent students from public and private schools \\ in Recife: ERICA
}

\author{
Myrtis Katille de Assunção Bezerra (https://orcid.org/0000-0002-4315-9623) ${ }^{1}$ \\ Eduardo Freese de Carvalho (https://orcid.org/0000-0002-8995-6089) ${ }^{1}$ \\ Juliana Souza Oliveira (https://orcid.org/0000-0003-1449-8930) ${ }^{2}$ \\ Eduarda Ângela Pessoa Cesse (https://orcid.org/0000-0001-5745-3981) ${ }^{1}$ \\ Pedro Israel Cabral de Lira (https://orcid.org/0000-0002-1534-1620) ${ }^{2}$ \\ Jonathan Galvão Tenório Cavalcante (https://orcid.org/0000-0003-4082-1092) ${ }^{3}$ \\ Vanessa Sá Leal (https://orcid.org/0000-0001-9492-2580) ${ }^{2}$ \\ Marcos Pereira Santos (https://orcid.org/0000-0003-3766-2502) ${ }^{4}$
}

\begin{abstract}
This study analyzes the behavior patterns related to the lifestyle of adolescents in Recife, Brazil. A cross-sectional study was conducted with data from the Cardiovascular Risk Study among Adolescents (ERICA) and 2400 students aged 12 to 17 were interviewed. The prevalence of physical activity, eating behavior, smoking and alcohol consumption were analyzed. The Chi-square test and 95\% confidence interval (95\%CI) were used to evaluate the statistical significance of the measurements. It was observed that $60 \%$ of the adolescents presented inadequate eating behaviors regarding the consumption of breakfast, daily water intake and meals eaten in front of the TV. About 40\% (95\%CI: 39.7-45) did not sit down to meals with their parents or guardians. The prevalence of physical inactivity was 52.4\% (95\%CI: 50-55). Female adolescents were more inactive (68.7\%, 95\%CI: 65-72) than males (36.4\%, 95\%CI: 3340). Teenagers of economic classes $D$ and $E$ were the most inactive (60.7\%, 95\%CI: 46-73). Alcohol consumption was $18.7 \%$ (95\%CI: 2.6-5.6) and smoking was prevalent in 3.8\% (95\% CI: 2-6) of the individuals. Inadequate eating behaviors and physical inactivity were the main areas responsible for an unhealthy lifestyle, followed by alcohol consumption and smoking.
\end{abstract}

Key words Smoking habit, Consumption of Alcoholic Beverages, Eating habits, Physical exercise
Resumo Este estudo analisa os comportamentos relacionados ao estilo de vida de adolescentes em Recife, Brasil. Estudo transversal com dados do Estudo de Riscos Cardiovasculares em Adolescentes (ERICA). 2.400 estudantes de 12 a 17 anos foram entrevistados. Foram analisadas as prevalências de atividade física, comportamento alimentar, tabagismo e consumo de álcool. $O$ teste Qui-quadrado e Intervalo de confiança 95\% (IC95\%) foram usados para avaliar a significância estatística das medidas. Observou-se 60\% dos adolescentes com comportamentos alimentares inadequados quanto ao consumo de café da manhã, água/dia e refeições em frente à $T V$. Cerca de 40\% (IC95\%: 39,7-45) não realizavam refeições com os pais ou responsáveis. A prevalência de inatividade física foi de 52,4\% (IC95\%: 50-55). Adolescentes do sexo feminino foram mais inativas (68,7\%; IC95\%: 65-72) em relação aos meninos (36,4\%; IC95\%: 33-40). Adolescentes das classes econômicas D e E foram os mais inativos (60,7\%; IC95\%: 46-73). O consumo de bebida alcoólica foi de 18,7\% (IC95\%: 2,6-5,6) e o tabagismo foi de 3,8\% (IC95\%: 2-6) dos indivíduos. Comportamentos alimentares inadequados e a inatividade física foram os principais domínios responsáveis por um estilo de vida não saudável, seguidos pelo consumo de álcool e tabagismo.

Palavras-chave Hábito de fumar, Consumo de Bebidas Alcoólicas, Hábitos Alimentares, Atividade física 


\section{Introdução}

As Doenças Crônicas Não Transmissíveis (DCNT) são a principal causa de morbimortalidade em populações de diferentes países e encontram-se associadas ao consumo de tabaco, consumo de alimentos ultraprocessados, sedentarismo e uso nocivo do álcool ${ }^{1-3}$. A adolescência, fase caracterizada por intensas mudanças biopsicossociais, constitui-se um dos períodos críticos para adoção desses comportamentos e, consequentemente, do início ou persistência de doenças crônicas, a exemplo da obesidade e do diabetes ${ }^{1-3}$. Os hábitos e conhecimentos adquiridos nesse período têm influência sobre muitos aspectos da vida adulta relacionados, por exemplo, à alimentação, prática de atividade física e saúde ${ }^{4,5}$. Logo, investigar o estilo de vida durante a adolescência, e em especial no ambiente escolar, é essencial para subsidiar estratégias de intervenção em saúde, no intuito de prevenir DCNTs na vida adulta.

Estudos epidemiológicos observacionais registram elevada ocorrência da exposição aos fatores de risco para DCNTs em adolescentes ${ }^{2,4}$. Uma dieta saudável é importante pois afeta o bem-estar e a saúde, e pode reduzir o risco de desenvolver doenças. No entanto, observa-se neste grupo etário comportamentos alimentares não saudáveis ${ }^{1,3,4}$.

O elevado consumo de bebidas açucaradas nessa fase é reconhecido como um dos fatores de risco para obesidade. Aumentar o consumo de água, associada ao menor consumo dessas bebidas, tem sido reportado como um importante fator de promoção da saúde e de diminuição da prevalência de excesso de peso entre crianças e adolescentes ${ }^{6}$.

Segundo a Pesquisa Nacional de Saúde de Estudantes (PeNSE) $7,64,4 \%$ dos estudantes brasileiros do $9^{\circ}$ ano tomaram café da manhã e 74,0\% almoçaram ou jantaram com os pais. O hábito de pular o café da manhã tem sido associado a menor concentração sérica de vitaminas e obesidade $^{8-10}$. Evidências sugerem que o consumo de café da manhã pode melhorar a função cognitiva relacionada à memória, notas de testes e frequência escolar ${ }^{11}$. Estudos confirmam que as refeições em família, incluindo o café da manhã, estão associadas a melhores comportamentos alimentares dos adolescentes e com a menor propensão ao excesso de peso ${ }^{10-12}$.

Dados da PeNSE registram que cerca de $60 \%$ dos estudantes brasileiros comem enquanto assistem televisão ${ }^{7}$. O consumo de alimentos em frente à tela, bem como o uso prolongado de mí- dia eletrônica tem sido relacionado à alimentação não saudável, com aumento do consumo fast foods, lanches e bebidas açucaradas ${ }^{13,14}$. O uso de mídia é conhecido por ser uma atividade de distração que pode reduzir a sensação de saciedade ao comer e que pode aumentar a propensão ao sobrepeso/obesidade ${ }^{14,15}$.

Um estudo realizado em escolares brasileiros mostra que apenas cerca de $40 \%$ deles são considerados suficientemente ativos ${ }^{7}$. A prática regular da atividade física e sua relação com benefícios físicos e psicológicos estão bem documentadas na literatura ${ }^{8,16}$. Assim como é reconhecido que a inatividade física nessa fase é um comportamento de risco que se associa à ocorrência de obesidade $^{16}$ e de DCNTs entre jovens ${ }^{1}$.

Outro problema refere-se ao uso de tabaco e álcool entre os adolescentes, diante do risco de dependência química e de doenças associadas. No Brasil, apesar de proibido o consumo por menores de idade, $18,5 \%$ dos adolescentes já fumaram pelo menos um cigarro ${ }^{17}$ e $21,2 \%$ referiram o consumo de bebidas alcoólicas ${ }^{18}$.

O conhecimento do estilo de vida de adolescentes constitui estratégia para o delineamento de ações de promoção da saúde, com intuito de prevenir doenças, as quais podem repercutir na saúde desse grupo ${ }^{19,20}$. Apesar da importância do tema, são poucos os estudos que avaliam o estilo de vida de adolescentes do nordeste brasileiro, $o$ que torna relevante conhecer os fatores comportamentais aos quais estão expostos.

Diante do risco aumentado de desenvolvimento de DCNTs em adolescentes ${ }^{1,2}$, torna-se necessário conhecer os fatores comportamentais aos quais estão expostos. Sendo assim, o objetivo desse estudo é analisar os comportamentos relacionados ao estilo de vida dos adolescentes no município de Recife, Brasil, de acordo com o cumprimento das recomendações para atividade física, comportamento alimentar, tabagismo e consumo de álcool.

\section{Métodos}

\section{População do estudo e amostragem}

Este trabalho faz parte do Estudo de Riscos Cardiovasculares em Adolescentes (ERICA). O ERICA é um estudo transversal, nacional e de base escolar que teve como objetivo estimar a prevalência de diabetes mellitus, obesidade, fatores de risco cardiovascular e de marcadores inflamatórios e de resistência à insulina em ado- 
lescentes de 12 a 17 anos matriculados em escolas públicas e privadas de cidades brasileiras com mais de 100 mil habitantes.

Para esse estudo foram selecionados os dados relativos ao município de Recife (PE). A coleta de dados do estudo ocorreu entre fevereiro de 2013 e novembro de 2014. As escolas foram selecionadas com probabilidade proporcional ao número de alunos da escola nos anos letivos considerados. A seleção amostral foi probabilística e estratificada em três estágios: escola, turma e alunos. As unidades amostrais foram as escolas e as turmas. As unidades de análise foram os adolescentes de 12 a 17 anos regularmente matriculados em escolas públicas ou privadas. Realizou-se o levantamento das turmas elegíveis $\left(7^{\circ}, 8^{\circ}\right.$ e $9^{\circ}$ ano do ensino fundamental e $1^{\circ}, 2^{\circ}$ e $3^{\circ}$ ano do ensino médio), das quais 03 foram sorteadas e todos seus alunos convidados para participar do estudo. $\mathrm{O}$ cálculo amostral foi realizado no programa estatístico Epi -info versão 7.1.5, considerando uma população infinita, baseou-se em uma prevalência de excesso de peso de $13,3 \%{ }^{21}$, um limite de confiança de $5 \%$, um efeito de desenho de 1,3 e nível de confiança de $95 \%$. O tamanho amostral mínimo foi de 2.301 adolescentes. No total foram selecionadas 29 escolas públicas, 10 escolas particulares e avaliados 2400 adolescentes, dos quais, $16,8 \%$ estavam com sobrepeso, 9,6\% com obesidade e 9\% hiperten$\operatorname{sos}^{20}$. Todos os estudantes das turmas selecionadas foram convidados a participar do estudo. Foram excluídos das análises apenas os adolescentes fora da faixa etária de 12 a 17 anos e adolescentes grávidas. Mais detalhes sobre a amostragem encontram-se em Vasconcelos et al. ${ }^{22}$.

\section{Coleta de dados}

Para coleta de dados foi utilizado um coletor eletrônico de dados, o personal digital assistant (PDA), que continha um questionário estruturado e autoaplicável com cerca de 100 questões distribuídas em blocos sobre características sociodemográficas, comportamento alimentar, prática de atividade física, tabagismo, consumo de bebida alcoólica e outros domínios.

\section{Características sociodemográficas}

Foram obtidas informações sobre sexo (masculino, feminino), nível socioeconômico e dependência administrativa da escola (pública ou privada). O nível socioeconômico foi definido pela utilização do Critério Brasil ${ }^{23}$, que considera posse de bens, presença de empregada doméstica e escolaridade do chefe da família. Este escore foi categorizado em níveis conforme recomendação do instrumento: A (35-46 pontos), B (23-34 pontos), C (14-22 pontos), D (8-13 pontos) e $\mathrm{E}$ (0-7 pontos). As classes $\mathrm{D}$ e $\mathrm{E}$ foram reagrupadas em uma mesma categoria devido a sua baixa frequência.

\section{Comportamento alimentar}

O bloco de comportamento alimentar incluiu a pergunta sobre o consumo diário de água, sendo consideradas como opções de resposta: "não bebe água", "bebe um a dois copos", "bebe três a quatro copos" e "bebe pelo menos cinco copos por dia”. Para análises a partir dessas respostas foram geradas duas categorias: " $<5$ copos" e " 5 copos ou mais de água”.

A questão sobre refeição na companhia dos pais ou responsáveis ${ }^{4}$ teve as seguintes opções de resposta: "às vezes”, "quase nunca faz uma ou duas refeições com pais ou responsáveis" e "quase sempre ou sempre faz pelo menos uma das refeições com os pais ou responsáveis". As respostas “às vezes” e "quase nunca” foram agrupadas para análise, formando a variável "às vezes ou quase nunca”.

Outro comportamento avaliado no mesmo questionário foi sobre realizar café da manhã, com as seguintes opções de resposta: "não", "às vezes", "quase sempre" e "sempre”. As respostas "quase sempre" e "sempre" foram agrupadas para a análise, obtendo-se a variável: "consome quase sempre/sempre”.

Foi questionado sobre almoçar e jantar assistindo TV e sobre comer petiscos (pipoca, biscoitos, salgadinhos, sanduíches, chocolates ou balas) assistindo TV, usando computador ou jogando videogame. No caso do almoço e do jantar, as alternativas de resposta foram: "nunca faz refeições em frente à TV”; “às vezes faz uma ou duas das refeições em frente à TV" e "faz quase sempre ou sempre faz pelo menos uma das refeições em frente à TV”. Para o consumo de petiscos nas condições listadas acima, as alternativas de resposta foram: "nunca come petisco em frente a telas"; "às vezes come petisco em frente a telas" e "quase sempre ou sempre come petisco em frente a telas”. Para fins de análise as repostas às vezes e nunca foram combinadas para gerar a categoria "às vezes ou nunca".

Para as análises, no bloco comportamento alimentar, foram considerados saudáveis a "ingestão de 5 copos ou mais de água/dia"; consumir café da manhã "quase sempre ou sempre"; realizar almoço e jantar com pais ou responsáveis "quase sempre ou sempre" ${ }^{4,6}$. Realizar refeições 
ou comer petiscos em frente à tela de vídeo game ou TV "às vezes ou nunca" também foram considerados comportamentos saudáveis ${ }^{4,9,15}$.

\section{Prática de atividade física}

Para determinação do nível de atividade física foi utilizada uma versão adaptada do Self-Administered Physical Activity Checklist ${ }^{24}$, o qual consiste em uma lista de 24 modalidades e permite que informe a frequência (dias) e o tempo (horas e minutos) que praticou, na última semana, alguma das atividades listadas. Os adolescentes que não acumularam pelo menos $300 \mathrm{~min} / \mathrm{semana}$ de atividade física foram considerados inativos no lazer ${ }^{25}$.

\section{Consumo de bebida alcoólica}

Para caracterização da ingestão de álcool, foi questionado aos adolescentes a idade em que tomou pelo menos um copo (ou dose) de bebida alcoólica pela primeira vez e dias de ingesta de pelo menos um copo (dose) de álcool, nos últimos 30 dias. Os indivíduos foram classificados quanto ao consumo (sim - ter consumido pelo menos 1 copo de bebida nos últimos 30 dias) ou não consumidor de bebida alcoólica. Foram classificados como não consumidores de bebidas alcoólicas os que responderam "nunca tomou bebida alcoólica" ou "nenhum dia nos últimos 30 dias" e foram considerados consumidores aqueles que responderam " 1 ou 2 dias", " 3 a 5 dias", " 6 a 9 dias", "10 a 19 dias", "20 a 29 dias" ou "todos os dias" nos últimos 30 dias. Foi perguntado também a idade em que tomou pelo menos um copo (ou dose) de bebida alcoólica pela primeira vez ${ }^{18}$.

\section{Tabagismo}

Neste estudo, definiu-se como "experimentação" ter provado ou fumado cigarros alguma vez na vida, mesmo uma ou duas tragadas. Foram considerados "fumantes atuais de cigarros" os jovens que fumaram cigarros pelo menos um dia nos últimos 30 dias. Ambas as variáveis seguiram as definições adotadas pela OMS e pelo Centro para Prevenção e Controle de Doenças dos Estados Unidos (Centers for Disease Control and Prevention - $\mathrm{CDC})^{26}$. Para investigar o uso frequente de tabaco, utilizou-se como indicador a reposta afirmativa para: "fumou cigarros por pelo menos sete dias seguidos". Além disso, os adolescentes informaram se eram expostos ou não à fumaça do tabaco de outras pessoas em casa.

\section{Análise Estatística}

As análises dos dados foram realizadas no software Stata versão 14.0, usando o módulo survey, que permite incorporar os aspectos referentes ao delineamento complexo da amostra, relacionada aos estratos, conglomerados e ponderações. Foram calculadas as estimativas de médias de consumo alimentar e de prevalência e intervalo de confiança de atividade física, comportamento alimentar e tabagismo e consumo de álcool, segundo as variáveis tipo de escola (pública e privada), sexo (masculino e feminino), classificação econômica (A e B, C, D e E). Os resultados da amostra foram expandidos para representar a população de escolares do município de Recife. O teste Qui-quadrado e o Intervalo de confiança 95\% (IC95\%) foram utilizados para avaliar a diferença das prevalências das variáveis de estilo de vida segundo as características da população estudada. O nível de significância estatística adotado foi de 5\%.

\section{Aspectos éticos}

O projeto deste estudo foi aprovado pela Comissão de ética da Universidade Federal de Pernambuco e os participantes assinaram o Termo de Consentimento Livre Esclarecido.

\section{Resultados}

Foram avaliados 2400 indivíduos cuja média de idade foi de 14,4 anos ( $D P=1,6)$ e $50,7 \%$ ( $\mathrm{n}=$ 1.113) eram do sexo masculino. Observou-se predominância das classes A e B em escolas privadas $(84,5 \%$ vs. $43,9 \%)$ e C $(51,4 \%$ vs. $10,0 \%)$ e $\mathrm{D}$ e $\mathrm{E}(3,1 \%$ vs. $0,4 \%)$ nas escolas públicas. $72,5 \%(n=1739)$ dos adolescentes procederam da rede pública de ensino.

No que se refere ao comportamento alimentar, o consumo de 5 copos ou mais de água por dia foi referido por $62,6 \%$ dos adolescentes ( Tabela 1). Observou-se que os adolescentes do sexo masculino apresentaram maior ingestão de água $(71,0 ;$ IC95\% $=67,9-73,9)$, quando comparados com as meninas $(53,9$; IC $95 \%=50,3-57,5)$, cuja diferença foi estatisticamente significante $(\mathrm{p}=<$ 0,001; Tabela 2).

Observou-se que 57,7\% (IC95\% = 55,1-60,2) realizavam sempre ou quase sempre refeições com os pais ou responsáveis (Tabela 1), o que foi mais predominante entre os adolescentes do sexo masculino (60\%; IC95\% = 56,8-63,2; $\mathrm{p}=0,013$; Tabela 2). 
Tabela 1. Descrição dos domínios de estilo de vida de adolescentes, segundo tipo de escola. Recife-PE, Brasil, 2013-2014 (População estimada = 142.421).

\begin{tabular}{|c|c|c|c|c|}
\hline \multirow[b]{2}{*}{ Domínios de estilo de vida } & \multirow{2}{*}{$\begin{array}{c}\text { Total de } \\
\text { Adolescentes \% } \\
\text { (IC95\%) }\end{array}$} & \multicolumn{2}{|c|}{ Tipo de escola } & \multirow[b]{2}{*}{$\mathbf{p}$} \\
\hline & & $\begin{array}{c}\text { Pública } \\
\% \text { (IC95\%) }\end{array}$ & $\begin{array}{c}\text { Privada } \\
\%(\text { IC95\%) }\end{array}$ & \\
\hline \multicolumn{5}{|l|}{ Comportamento alimentar } \\
\hline \multicolumn{5}{|l|}{ Consumo diário de água } \\
\hline$<5$ copos & $37,3(34,8-39,9)$ & $37,4(34,7-40,1)$ & $37,2(32,0-42,6)$ & \\
\hline 5 copos ou mais & $62,8(60,0-65,1)$ & $62,5(59,8-65,2)$ & $62,8(57,3-67,9)$ & 0,931 \\
\hline \multicolumn{5}{|l|}{ Consome café da manhã } \\
\hline Nunca ou quase nunca & $19,3(16,8-22,0)$ & $18,9(15,8-22,5)$ & $20,3(18,0-22,8)$ & \\
\hline Às vezes & $32,7(29,7-35,7)$ & $35,5(32,8-38,2)$ & $25,0(20,4-30,3)$ & \\
\hline Quase sempre ou sempre & $47,9(44,2-51,6)$ & $45,5(41,7-49,4)$ & $54,6(48,4-60,6)$ & $0,002^{\star}$ \\
\hline \multicolumn{5}{|l|}{ Petiscar em frente à tela } \\
\hline Às vezes ou nunca & $55,8(52,8-58,7)$ & $56,2(53,3-59,1)$ & $54,6(46,7-62,3)$ & \\
\hline Quase sempre ou sempre & $44,2(41,2-47,2)$ & $43,7(6,1-9,8)$ & $45,3(37,7-53,2)$ & 0,692 \\
\hline \multicolumn{5}{|l|}{ Refeições em frente à tela } \\
\hline Às vezes ou nunca & $40,0(38,6-43,3)$ & $40,5(38,0-43,0)$ & $42,1(36,7-46,7)$ & \\
\hline Quase sempre ou sempre & $59,0(56,6-61,3)$ & $59,4(56,9-61,9)$ & $57,8(52,2-63,2)$ & 0,590 \\
\hline \multicolumn{5}{|l|}{ Refeições com a família } \\
\hline Às vezes ou nunca & $42,2(39,7-44,8)$ & $43,7(40,3-47,2)$ & $38,1(33,6-42,9)$ & \\
\hline Quase sempre ou sempre & $57,7(55,1-60,2)$ & $52,2(52,7-59,6)$ & $61,8(57,0-66,3)$ & 0,07 \\
\hline \multicolumn{5}{|l|}{ Atividade Física/semana } \\
\hline Ativo & $47,6(45,0-50,2)$ & $47,2(43,9-50,4)$ & $48,9(43,9-53,9)$ & 0,574 \\
\hline Insuficientemente ativo & $52,4(49,7-54,9)$ & $52,8(49,6-56,5)$ & $51,1(46,1-56,0)$ & \\
\hline \multicolumn{5}{|l|}{ Tabagismo atual } \\
\hline Não & $96,2(94,3-97,3)$ & $95,5(93,5-4,4)$ & $97,6(95,2-98,8)$ & \\
\hline Sim & $3,8(2,6-5,6)$ & $4,4(2,9-6,4)$ & $2,3(1,1-4,7)$ & 0,104 \\
\hline \multicolumn{5}{|l|}{ Consumo de álcool } \\
\hline Não & $81,2(78,3-83,7)$ & $81,4(78,2-84,1)$ & $80,7(78,3-83,7)$ & \\
\hline Sim & $18,7(16,2-21,6)$ & $18,6(15,8-21,7)$ & $19,2(13,6-26,5)$ & 0,847 \\
\hline
\end{tabular}

Realizar o café da manhã quase sempre ou sempre foi o único comportamento com diferença estatisticamente significante, sendo mais prevalente em estudantes de escolas privadas $(54,6 \%$; IC95\% = 48,4-60,6) do que por adolescentes da rede pública de ensino (45,5\%; IC95\% $=41,7-49,4)(\mathrm{p}=<0,01 ;$ Tabela 1$)$, e menos frequente em adolescentes do sexo feminino $(41,2 \%$; IC95\% $=36,9-45,7)$ que entre os meninos $(54,5 \%$; IC95\% = 50,2-58,7) $(\mathrm{p}=<0,001$; Tabela 2).

Cerca da metade dos adolescentes consumiam petiscos quase sempre ou sempre em frente à tela. O relato de quase sempre ou sempre realizar as refeições (almoço ou jantar) em frente à TV foi registrado em 59,0\% (IC95\% = 56,6-61,3) dos adolescentes.

A prevalência de atividade física insuficiente na semana foi de 52,4\% (IC95\% = 49,7-54,9) en- tre os adolescentes (Tabela 1). Não houve diferença estatisticamente significante entre adolescentes ativos de escolas públicas e privadas (Tabela 1). Porém, adolescentes do sexo feminino foram mais insuficientemente ativas (68,7\%; IC95\% $=65,0-72,3)$ do que aqueles do sexo masculino $(36,4 \%$; IC95\% = 33,0-39,9) ( $\mathrm{p}=<0,001$; Tabela 2). Adolescentes das classes econômicas D e E, também foram mais inativos $(60,7 \%$, IC $95 \%=$ $46,4-73,4)$ que os da classe econômica A $(47,3 \%$, IC95\% $=43,5-51,2)(\mathrm{p}=0,012$; Tabela 3$)$.

No presente estudo, 51,6\% (IC95\% = 48,0$56,2)$ dos adolescentes nunca experimentaram bebida alcoólica, 7,5\% experimentaram álcool pela primeira vez entre os 9 e 11 anos e $34,2 \%$ entre os 12 e 17 anos de idade. A Tabela 1 mostra que $18,7 \%$ (IC95\% = 16,2-21,6) consumiram bebida alcóolica pelo menos uma vez nos últimos 30 dias. Não foi observada diferença estatistica- 


\begin{tabular}{|c|c|c|c|c|}
\hline 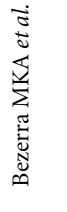 & $\begin{array}{l}\text { Tabela 2. Descrição dos domínios de esti } \\
\text { (População estimada = 142.421). }\end{array}$ & adolescentes, segu & o sexo. Recife-PE, $\mathrm{I}$ & 2013-2014 \\
\hline & & & & \\
\hline & Domínios de estilo de vida & $\begin{array}{l}\text { Feminino } \\
\%(\text { IC95\%) }\end{array}$ & $\begin{array}{l}\text { Masculino } \\
\%(\text { IC95\%) }\end{array}$ & $\mathbf{p}$ \\
\hline & Comportamento alimentar & & & \\
\hline & Consumo diário de água & & & \\
\hline & $<5$ copos & $46,0(42,5-49,6)$ & $28,9(26,0-32,0)$ & \\
\hline & 5 copos ou mais & $53,9(50,3-57,5)$ & $71,0(67,9-73,9)$ & $<0,001^{\star}$ \\
\hline & Consume de café da manhã & & & \\
\hline & Nunca ou quase nunca & $23,2(19,2-27,7)$ & $15,5(12,8-18,6)$ & \\
\hline & Às vezes & $35,5(31,1-40,1)$ & $29,9(26,1-34,1)$ & \\
\hline & Quase sempre ou sempre & $41,2(36,9-45,7)$ & $54,5(50,2-58,7)$ & $<0,001^{\star}$ \\
\hline & Petiscar em frente à tela & & & \\
\hline & Às vezes ou nunca & $55,2(51,6-58,8)$ & $56,3(52,2-60,3)$ & \\
\hline & Quase sempre ou sempre & $44,7(41,1-48,3)$ & $43,6(39,6-47,7)$ & 0,667 \\
\hline & Refeições em frente à tela & & & \\
\hline & Às vezes ou nunca & $42,4(38,6-46,4)$ & $39,5(35,9-43,2)$ & \\
\hline & Quase sempre ou sempre & $57,5(53,5-61,4)$ & $60,5(56,8-64,0)$ & 0,315 \\
\hline & Refeições com a família & & & \\
\hline & Às vezes ou quase nunca & $44,7(41,5-47,8)$ & $39,9(36,8-43,1)$ & \\
\hline & Quase sempre ou sempre & $55,3(52,1-58,4)$ & $60,0(56,8-63,2)$ & $0,013^{\star}$ \\
\hline & Atividade Física/semana & & & \\
\hline & Ativo & $31,2(27,7-34,9)$ & $63,5(60,0-66,9)$ & \\
\hline & Insuficientemente ativo & $68,7(65,0-72,3)$ & $36,4(33,0-39,9)$ & $<0,001^{\star}$ \\
\hline & Tabagismo atual & & & \\
\hline & Não & $96,4(93,9-97,9)$ & $95,8(94,1-97,0)$ & \\
\hline & Sim & $3,5(2,0-6,1)$ & $4,1(2.9-5,8)$ & 0,491 \\
\hline & Consumo de bebida alcoólica & & & \\
\hline & Não & $82,5(78,6-85,8)$ & $79,9(76,2-83,1)$ & \\
\hline & Sim & $17,4(14,1-21,3)$ & $20,0(16,8-23,8)$ & 0,259 \\
\hline
\end{tabular}

mente significante no consumo de bebidas alcóolicas de acordo com sexo, rede de ensino e classificação econômica (Tabelas 1,2,3).

No momento da pesquisa 3,8\% (IC95\% = 2,6-5,6) dos adolescentes eram tabagistas atuais (Tabela 1), 17,0 \% (IC95\% = 14,3-19,7) experimentaram ou tentaram fumar pelo menos 1 vez na vida, $1,3 \%($ IC95\% $=0,8-1,8)$ fumaram cigarros por pelo menos 7 dias seguidos e $29,6 \%$ (IC95\% $=26,3-32,9)$ tinham contato com fumaça de cigarros de outros tabagistas em casa.

\section{Discussão}

Os domínios de estilo de vida mais prevalentes entre adolescentes foram o comportamento alimentar inadequado e a inatividade física, segui- dos pelo consumo de bebida alcoólica e pelo tabagismo. A distribuição desses comportamentos revelou padrões distintos entre sexo (masculino e feminino), entre as classes sociais mais altas e mais baixas e por tipo de escola (pública e privada).

É reconhecido que ações promotoras de saúde no ambiente escolar podem promover estilos de vida saudáveis ${ }^{20,27}$. No entanto, em Recife (PE), a carência de apoio do setor saúde nas escolas visando ações que promovam a alimentação saudável nesse ambiente, e para essa faixa etária, assim como a ausência de locais com estrutura física adequada para a prática de esportes em escolas públicas ${ }^{19}$, pode justificar o atual quadro de adolescentes com comportamentos alimentares inadequados e insuficientemente ativos. No estudo realizado em São Paulo, Brasil, apesar de 
Tabela 3. Descrição dos domínios de estilo de vida de adolescentes, segundo nível econômico. Recife-PE, Brasil, 2013-2014 (População estimada = 142.421).

\begin{tabular}{|c|c|c|c|c|}
\hline \multirow[b]{2}{*}{ Domínios de estilo de vida } & \multicolumn{3}{|c|}{ Classificação econômica } & \multirow[b]{2}{*}{$\mathbf{p}$} \\
\hline & $\begin{array}{c}\text { A e B } \\
\%(\text { IC95\%) }\end{array}$ & $\begin{array}{c}\mathrm{C} \\
\%(\mathrm{IC} 95 \%)\end{array}$ & $\begin{array}{c}\text { D e E } \\
\%(\text { IC95\%) }\end{array}$ & \\
\hline \multicolumn{5}{|l|}{ Comportamento alimentar } \\
\hline \multicolumn{5}{|l|}{ Consumo diário de água } \\
\hline$<5$ copos & $37,0(33,4-40,8)$ & $37,2(31,8-42,9)$ & $25,1(15,4-38,0)$ & \\
\hline 5 copos ou mais & $62,9(59,1-66,5)$ & $62,7(57,0-68,1)$ & $74,8(61,9-84,5)$ & 0,330 \\
\hline \multicolumn{5}{|l|}{ Consume café da manhã } \\
\hline Nunca ou quase nunca & $19,3(16,4-22,6)$ & $15,7(12,8-19,1)$ & $22,3(12,8-35,9)$ & \\
\hline Às vezes & $28,6(25,1-32,4)$ & $36,8(30,7-43,3)$ & $38,4(22,7-5)$ & \\
\hline Quase sempre ou sempre & $52(47,9-56,8)$ & $47,4(41,8-53,1)$ & $39,2(22,8-58,3)$ & 0,08 \\
\hline \multicolumn{5}{|l|}{ Petiscar em frente à tela } \\
\hline Às vezes ou nunca & $55,1(50,7-59,3)$ & $56,1(50,7-61,4)$ & $59,7(32,5-82,0)$ & \\
\hline Quase sempre ou sempre & $44,9(40,6-49,2)$ & $43,8(38,5-49,2)$ & $40,2(17,9-67,4)$ & 0,873 \\
\hline \multicolumn{5}{|l|}{ Refeições em frente à tela } \\
\hline Às vezes ou nunca & $41,9(37,9-46,1)$ & $39,0(34,3-44,0)$ & $42,4(29,4-56,4)$ & \\
\hline Quase sempre ou sempre & $58,0(53,8-62,0)$ & $60,9(55,9-65,7)$ & $57,6(43,5-70,5)$ & 0,575 \\
\hline \multicolumn{5}{|l|}{ Refeições com a família } \\
\hline Às vezes ou quase nunca & $41,7(37,6-58,2)$ & $40,9(35,4-46,7)$ & $44,3(26,6-63,6)$ & \\
\hline Quase sempre ou sempre & $58,2(55,0-62,3)$ & $59,0(53,2-64,5)$ & $55,6(36,3-73,3)$ & 0,918 \\
\hline \multicolumn{5}{|l|}{ Atividade Física/semana } \\
\hline Ativo & $52,6(48,7-56,4)$ & $43,3(37,6-49,3)$ & $39,2(26,5-53,5)$ & \\
\hline Insuficientemente ativo & $47,3(43,5-51,2)$ & $56,6(50,7-62,3)$ & $60,7(46,4-73,4)$ & $0,012^{\star}$ \\
\hline \multicolumn{5}{|l|}{ Tabagismo atual } \\
\hline Não & $97,0(95,1-98,2)$ & $95,6(92,8-97,4)$ & $97,3(82,3-99,6)$ & \\
\hline $\operatorname{Sim}$ & $2,9(1,7-4,8)$ & $4,3(2,5-7,1)$ & $2,6(0,3-17,6)$ & 0,363 \\
\hline \multicolumn{5}{|l|}{ Consumo de álcool } \\
\hline Não & $79,0(75,1-82,4)$ & $83,1(78,1-87,1)$ & $77,9(66,1-86,5)$ & \\
\hline Sim & $20,9(17,5-24,8)$ & $16,8(12,8-21,8)$ & $22,0(13,4-33,9)$ & 0,183 \\
\hline
\end{tabular}

ter sido utilizada uma metodologia diferente, foi observado que o consumo alimentar inadequado também foi o principal domínio responsável pelo estilo de vida não saudável neste grupo ${ }^{5}$. Essas pesquisas apontam que mais ações são necessárias para os adolescentes, os quais ainda são um grupo negligenciado pelo setor saúde.

No presente estudo, 62,6\% dos adolescentes de Recife consumiam 5 ou mais copos de água, prevalência maior que a nacional $(48,2 \%)^{4}$. Estudos realizados no Brasil apontam que o maior consumo de água foi mais observado nas regiões Norte, Nordeste e Centro-Oeste e menor nas regiões Sudeste e Sul ${ }^{4,7}$, o que pode explicar essa diferença entre as regiões é, principalmente, o clima que favorece o maior ou menor consumo. No entanto, é importante destacar que cerca de $40 \%$ dos adolescentes de Recife informaram um baixo consumo ( $<5$ copos/dia). A ingestão de água é considerada uma prática de saúde, visto que sua falta (desidratação) pode influenciar na concentração, vigilância e memória de curto prazo e ocasionar problemas como cefaleia e litíase urinária $^{28}$. Além disso, a ingestão adequada tem sido associada ao menor consumo energético, o que pode contribuir na prevenção da obesidade e síndrome metabólica ${ }^{6,29}$.

A realização das refeições com os pais foi um comportamento referido por mais da metade dos adolescentes de Recife (57,7\%). Prevalência menor que a demonstrada na Pesquisa Nacional de Saúde do Escolar (PeNSE) $(62,6 \%)^{3}$. Realizar as refeições em família é um importante aspecto do ambiente familiar que pode promover comportamentos alimentares saudáveis na adolescência e percepção corporal positiva ${ }^{9,10}$, assim como 
participar de atividades conjuntas exercem efeito protetor na prevenção de uso de álcool e drogas ${ }^{30}$.

No que se refere à omissão do café da manhã, este domínio foi mais prevalente em estudantes de escolas públicas $(23,2 \%)$ do que em estudantes de escolas privadas (15,5\%). Dados da PeNSE reportam que cerca de $40 \%$ dos estudantes brasileiros referiram ter omitido essa refeição e que esse comportamento foi mais prevalente em estudante de escolas privadas ${ }^{7}$. O hábito de tomar café da manhã tem sido associado ao baixo risco de sobrepeso e obesidade ${ }^{9,10,31}$ e de deficiências nutricionais ${ }^{8}$, bem como à melhoria na capacidade de aprendizagem e de comportamentos alimentares mais saudáveis ${ }^{11,12}$. Vários são os fatores que têm interferido nesse comportamento. Em adolescentes, destacam-se a preocupação com a imagem corporal, o desejo de fazer dieta para perder peso, a pouca disponibilidade de tempo para realizar as refeições e a redução de fome nesse momento do dia ${ }^{32}$. Porém, essa maior prevalência de não consumo de café da manhã por estudantes de escolas públicas pode ter ocorrido pelo menor acesso aos alimentos, consequente da menor condição socioeconômica.

Cerca de metade dos adolescentes tinha o hábito de realizar quase sempre ou sempre as refeições ou o consumo de petiscos em frente à tela. Essa é considerada uma prática de risco, visto que as propagandas alimentares e/ou programas de televisão provocam distração que podem promover mudanças neurofisiológicas e interferir nos sinais de fome e saciedade ${ }^{33}$. Diante da crescente exposição às telas e dos danos à saúde que estas podem causar, preconiza-se que o tempo dispendido em frente à TV pelos jovens não deva ultrapassar $1 \mathrm{~h}$ a $2 \mathrm{~h}$ por dia ${ }^{34}$. Apesar do difícil controle do tempo de tela entre adolescentes, em revisão sistemática, Schmidt et al. ${ }^{35}$ constataram que, na maioria dos estudos, intervenções no âmbito escolar mostraram resultados positivos em promover a redução do tempo dispendido em frente às telas.

No que se refere à prática de atividade física, foi encontrada uma prevalência duas vezes maior em adolescentes do sexo masculino $(63,5 \%$ vs $31,2 \%)$ e mais prevalente nos estudantes de classe econômica de maior renda ( $60,7 \%$ vs $39,7 \%)$, conforme previamente descrito em outros estu$\operatorname{dos}^{36,37}$. Ao avaliarem as barreiras percebidas para prática de atividade física, Dias et al. ${ }^{37}$ descrevem que a falta de companhia $(75,8 \%)$, a preferência por outras atividades $(64,1 \%)$ e o clima $(54,9 \%)$ foram as mais reportadas por adolescentes do sexo feminino. Para Farias Júnior et al. ${ }^{24}$ os maio- res níveis de atividade física no sexo masculino podem ser explicados por diferenças biológicas, socioculturais, de percepção de corpo e atributos de gênero. Já a inatividade física em adolescentes de menor poder aquisitivo justifica-se pela insuficiência de locais públicos seguros e com estrutura de qualidade, ao passo que aqueles oriundos de famílias com maior renda teriam acesso a espaços públicos e privados de melhor qualidade ${ }^{37}$. Logo, a desigualdade social pode contribuir para a inatividade física.

Os resultados desse levantamento indicam que a proporção de adolescentes expostos ao consumo de bebidas alcoólicas (18,7\%) foi inferior tanto em comparação aos dados de uma pesquisa nacional com escolares $(27,3 \%)^{38}$ quanto ao estudo realizado com 4.207 estudantes do ensino médio da rede pública $(30,4 \%)$ da região metropolitana, zona da mata, agreste, sertão e sertão do São Francisco de Pernambuco ${ }^{39}$. O consumo de bebidas alcoólicas pode trazer consequências à saúde dos adolescentes, aumentando o risco de acidentes de trânsito, ferimentos não intencionais, homicídio e suicídio, os quais representam a maior causa de morte entre joven $s^{1,40}$. Em 2012, estimou-se que 5,9\% (3,3 milhões) de todas as mortes no mundo foram associadas ao consumo de álcool ${ }^{40}$.

A prevalência de tabagismo encontrado em adolescentes de Recife também apresentou resultado inferior ao descrito na pesquisa realizada por Bezerra et al. ${ }^{39}(7,6 \%)$, com estudantes da rede pública de ensino do interior e região metropolitana de Pernambuco. O maior número de tabagistas descritos nesse estudo justifica-se pela frequência de fumantes de cigarro ser maior no interior do que nas capitais brasileiras ${ }^{7}$.

A exposição à fumaça de cigarros tanto a curto quanto em longo prazo pode apresentar consequências respiratórias e não respiratórias, bem como dependência de nicotina $\mathrm{a}^{40}$. O contato com fumaça de cigarros de outros fumantes em casa foi referido por mais de 1/4 dos adolescentes do Recife. Crianças e adolescentes quando expostas ao tabagismo dos pais apresentam comprometimento da saúde vascular até 25 anos mais tarde. Nesse sentido, deve haver esforços contínuos para reduzir o tabagismo entre adultos e adolescentes para proteger os jovens e reduzir a carga de doenças cardiovasculares em toda a população $0^{41}$. A escola é considerada um dos ambientes propícios para o desenvolvimento de ações educativas com adolescentes e sua família, a parceria com o setor saúde, nesse cenário, é essencial para estratégias mais efetivas ${ }^{19,20,27}$. 
As ações direcionadas aos adolescentes podem utilizar a escola como ambiente promotor de estilo de vida saudável, com apoio da comunidade e do setor saúde para incentivar escolhas alimentares saudáveis por meio de educação alimentar e nutricional, apoiar a prática de atividade física e desenvolver ações de sensibilização sobre os riscos do uso do tabaco e do álcool à saúde. A redução da carga global das DCNTs através dessas políticas é uma prioridade e uma condição necessária para a efetiva mudança no perfil epidemiológico desse grupo.

O presente estudo, por possuir delineamento transversal não possibilita extrapolações sobre fatores causais. Apesar disso, considerando o tamanho amostral e a representatividade do ERICA, traz uma importante contribuição para o mapeamento do estilo de vida dos adolescentes do município do Recife.

Com base no exposto, os adolescentes de Recife apresentam uma baixa frequência de comportamentos alimentares de proteção à saúde $\mathrm{e}$ de prática regular de atividade física. Podemos concluir que ações que promovam esses comportamentos devam ser implementadas. Essas ações devem considerar que a menor condição socioeconômica está associada a menor prática de atividade física. Outra questão a ser destacada refere-se à elevada prevalência de adolescentes que consomem bebida alcóolica, tabagistas e os que entram em contato com fumaça de cigarros de outros tabagistas em casa e, consequentemente, estão expostos aos riscos associados ao fumo passivo e ao consumo de álcool precoce.

As políticas destinadas ao incentivo da promoção da saúde, voltadas à redução da inatividade física, do comportamento alimentar inadequado, do tabagismo e do consumo de álcool, no ambiente escolar, devem ser intersetoriais e multidisciplinares. A utilização de abordagens educativas e pedagógicas que privilegiem os processos ativos e incorporem os conhecimentos e a participação da família e da comunidade é essencial na promoção da saúde deste grupo etário.

\section{Colaboradores}

Planejamento do estudo: MKA Bezerra, JS Oliveira, PIC Lira, VS Leal. Análise e interpretação dos dados: MKA Bezerra, EF Carvalho, EAP Cesse, MP Santos, JS Oliveira. Redação do artigo: MKA Bezerra, EF Carvalho, JS Oliveira, EAP Cesse, PIC Lira, JGT Cavalcante, VS Leal, MP Santos. Todos os autores aprovaram a versão final do artigo. 


\section{Financiamento}

Departamento de Ciência e Tecnologia da Secretaria de Ciência, Tecnologia e Insumos Estratégicos do Ministério da Saúde (Decit/SCTIE/MS), Fundo Setorial de Saúde (CT-Saúde) do Ministério da Ciência, Tecnologia e Inovação (MCTI).

\section{Agradecimento}

Ao Instituto Brasileiro de Geografia e Estatística pela cessão dos coletores eletrônicos de dados, o PDA (personal digital assistant).

\section{Referências}

1. World Health Organization (WHO). Global status report on noncommunicable diseases 2014. Geneva: WHO: 2014.

2. Lavrador MSF, Abbes PT, Escrivão MAMS, Taddei JAAC. Riscos cardiovasculares em adolescentes com diferentes graus de obesidade. Arq Bras Cardiol 2011; 96(3):205-211.

3. Levy RB, Castro IRR, Cardoso LO, Tavares LF, Sardinha LMV, Gomes FS, Costa AWN. Consumo e comportamento alimentar entre adolescentes brasileiros: Pesquisa Nacional de Saúde do Escolar (PeNSE), 2009. Cien Saude Colet 2010; 15(Supl. 2):3085-3097.

4. Barufaldi LA, Abreu GA, Oliveira JS, Santos DF, Fujimori E, Vasconcelos SML, Vasconcelos FAG, Tavares BM. ERICA: prevalência de comportamentos alimentares saudáveis em adolescentes brasileiros. Rev Saude Publica 2016; 50(Supl. 1):6s.

5. Ferrari TK, Cesar CLG, Alves MCGP, Barros MBA, Goldbaum M, Fisberg RM. Estilo de vida saudável em São Paulo, Brasil. Cad Saúde Pública 2017; 33(1):e00188015.

6. Schwartz AE, Leardo M, Aneja S, Elbel B. Effect of a School-Based Water Intervention on Child Body Mass Index and Obesity. JAMA Pediatr 2016; 170(3):220226.

7. Instituto Brasileiro de Geografia e Estatística (IBGE). Pesquisa nacional de saúde da escola: 2015. Coordenação de População e Indicadores Sociais. Rio de Janeiro: IBGE; 2016.

8. Mielgo-Ayuso J, Valtueña J, Cuenca-García M, Gottrand F, Breidenassel C, Ferrari M, Manios Y, De Henauw S, Widhalm K, Kafatos A, Kersting M, Huybrechts I, Moreno LA, González-Gross M, HELENA Study Group. Regular breakfast consumption is associated with higher blood vitamin status in adolescents: the HELENA (Healthy Lifestyle in Europe by Nutrition in Adolescence) Study. Public Health Nutr 2017; 20(8):1393-1404.

9. Larson N, MacLehose R, Fulkerson JA, Berge JM, Story M, Neumark-Sztainer D. Eating breakfast and dinner together as a family: associations with sociodemographic characteristics and implications for diet quality and weight status. J Acad Nutr Diet 2013; 113:1601-1609.

10. Marques A, Naia A, Branquinho C, Matos MG. Adolescents' eating behaviors and its relationship with family meals, body mass index and body weight perception. Nutr Hosp 2018; 35:550-556.

11. Rampersaud GC, Pereira MA, Girard BL, Adams J, Metzl J. Breakfast habits, nutritional status, body. weight, and academic performance in children and adolescents. J Am Diet Assoc 2005; 105(5):743-760.

12. Nansel TR, Haynie DL, Lipsky LM, Wang J, Mehta SN, Laffel LM. Relationships among parent and youth healthful eating attitudes and youth dietary intake in a cross-sectional study of youth with type 1 diabetes. Int J Behav Nutr Phys Act 2013; 10:125.

13. Sampasa-Kanyinga H, Chaput J-P, Hamilton HA. Associations between the use of social networking sites and unhealthy eating behaviours and excess body weight in adolescents. Br J Nutr 2015; 114(11):19411947. 
14. Mittal D, Stevenson RJ, Oaten MJ, Miller LA. Snacking while watching TV impairs food recall and promotes food intake on a later TV free test meal. Appl Cogn Psychol 2011; 25(6):871-877.

15. Cha EM, Hoelscher DM, Ranjit N, Chen B, Gabriel KP, Kelder S, Saxton DL. Effect of Media Use on Adolescent Body Weight. Prev Chronic Dis 2018; 15:E141.

16. Souza CO, Silva RCR, Assis AMO, Fiaccone RL, Pinto EJ, Moraes LTLP. Associação entre inatividade física e excesso de peso em adolescentes de Salvador, Bahia Brasil. Rev Bras Epidemiol 2010; 13(3):468-475.

17. Figueiredo VC, Szklo AS, Costa LC, Kuschnir MCC, Silva TLN, Bloch KV, Szklo M. ERICA: prevalência de tabagismo em adolescentes brasileiros. Rev Saúde Pública 2016; 50(Supl. 1):12s.

18. Coutinho ESF, França-Santos D, Magliano ES, Bloch KV, Barufaldi LA, Cunha CF, Vasconcellos MTL, Szklo M. ERICA: padrões de consumo de bebidas alcoólicas em adolescentes brasileiros. Rev Saude Publica 2016; 50(Supl. 1):8s.

19. Bezerra MA, Carvalho EF, Oliveira JS, Leal VS. Saúde e nutrição em escolas públicas e privadas de Recife. Rev Bras Saude Mater Infant 2017; 17(1):191-200.

20. Bezerra MKA, Carvalho EF, Oliveira JS, Cesse EAP, Lira PIC, Cavalcante JGT, Leal VS. Health promotion initiatives at school related to overweight, insulin resistance, hypertension and dyslipidemia in adolescents: a cross-sectional study in Recife, Brazil. BMC Public Health 2018; 18:223.

21. Leal VS, Lira PIC, Oliveira JS, Menezes RCE, Sequeira LAS, Arruda Neto MA, Andrade SLLS, Batista Filho M. Excesso de peso em crianças e adolescentes no Estado de Pernambuco, Brasil: prevalência e determinantes. Cad Saude Publica 2012; 28(6):1175-1182.

22. Vasconcellos MTL, Silva PLN, Szklo M, Kuschnir MCC, Klein CH, Abreu GA, Barufaldi LA, Bloch KV. Desenho da amostra do Estudo do Risco cardiovascular em Adolescentes (ERICA). Cad Saude Publica 2015; 31(5):921-930.

23. Associação Brasileira de Empresas de Pesquisa (ABEP). Critério Brasil de avaliação econômica 2013. São Paulo: ABEP; 2013.

24. Farias Júnior JC, Lopes AS, Mota J, Santos MP, Ribeiro JC, Hallal PC. Validade e reprodutibilidade de um questionário para medida de atividade física em adolescentes: uma adaptação do Self-Administered Physical Activity Checklist. Rev Bras Epidemiol 2012; 15(1):198-210

25. World Health Organization (WHO). Global recommendations on physical activity for health. Geneva: WHO; 2010.

26. Warren CW, Jones NR, Peruga A, Chauvin J, Baptiste JP, Silva VC, Awa F, Tsouros A, Rahman K, Fishburn B, Bettcher DW, Asma S, Centers for Disease Control and Prevention (CDC). Global youth tobacco surveillance, 2000-2007. MMWR Surveill Summ 2008; 57(1):1-28.
27. Langford R, Bonell CP, Jones HE, Pouliou T, Murphy SM, Waters E, Komro KA, Gibbs LF, Magnus D, Campbell R. The world health organization's health promoting schools framework: a cochrane systematic review and meta-analysis. BMC Public Health 2015; 15:130.

28. Popkin B, D'Anci K, Rosenberg I. Water, hydration and health. Nutr Rev 2010; 68(8):439-458.

29. Tobarra MM, García-Hermoso A, Lahoz-García N, Notario-Pacheco B, Lucas-de la Cruz L, Pozuelo-Carrascosa DP, García-Meseguer MJ, Martínez-Vizcaíno V. The association between water intake, body composition and cardiometabolic factors among children - The Cuenca study. Nutr Hosp 2016; 33(Supl. 3):1926.

30. Guimarães ABP, Hochgraf PB, Brasiliano S, Ingberman YK. Aspectos familiares de meninas adolescentes dependentes de álcool e drogas. Rev psiquiatr clín 2009; 36(2):69-74.

31. Cnop ML, Monteiro LS, Rodrigues PRM, Estima CCP, Veiga GV, Pereira RA. Meal habits and anthropometric indicators in adolescents from public and private schools of the metropolitan region of Rio de Janeiro. Rev Nutr 2018; 31(1):35-47.

32. Trancoso SC, Cavalli SB, Proença RPC. Café da manhã: caracterização, consumo e importância para a saúde. Rev Nutr 2010; 23(5):859-869.

33. Spence C, Okajima K, Cheok AD, Petit O, Michel C. Eating with our eyes: From visual hunger to digital satiation. Brain Cognition 2016; 110:53-63.

34. American Academy of Pediatrics, Committee on Public Education. Children, adolescents, and television. Pediatrics 2001; 107(2):423-426.

35. Schmidt ME, Haines J, O'Brien A, McDonald J, Price S, Sherry B, Taveras EM. Systematic review of effective strategies for reducing screen time among young children. Obesity 2012; 20(7):1338-1354.

36. Cureau FV, Silva TLN, Bloch KV, Fujimori E, Belfort DR, Carvalho KMB, Leon EB, Vasconcellos MTL, Ekelund U, Schaan BD. ERICA: inatividade física no lazer em adolescentes brasileiros. Rev Saude Publica. 2016; 50(Supl. 1):4s.

37. Dias DF, Loch MR, Ronque ERV. Barreiras percebidas à prática de atividades físicas no lazer e fatores associados em adolescentes. Cien Saude Colet 2015; 20(11):3339-3350.

38. Malta DC, Porto DL, Melo FCM, Monteiro RA, Sardinha LMV, Lessa BH. Família e proteção ao uso de tabaco, álcool e drogas em adolescentes, Pesquisa Nacional de Saúde dos Escolares. Rev Bras Epidemiol 2011; 14(Supl. 1):166-177.

39. Bezerra J, Lopes AS, Hardmam CM, Tassitano RM, Tenório MCM, Barros MVG. Consumo de bebidas alcoólicas e tabagismo: associação com inatividade física no lazer e comportamento sedentário. Rev Andal Med Deporte 2015; 8(1):1-6. 
40. Currie C, Zanotti C, Morgan A, Currie D, Looze M, Roberts C, Samdal O, Smith ORF, Barnekow V. Social determinants of health and well-being among young people. Health Behaviour in School-aged Children (HBSC) Study: international report from the 2009/2010 survey. Copenhagen: WHO Regional Office for Europe; 2012.

41. United States Public Health Service. Office of the Surgeon General. The Health Consequences of Smoking-50 Years of Progress: A Report of the Surgeon General: Executive Summary. Rockville: U.S. Dept. of Health and Human Services, Public Health Service, Office of the Surgeon General; 2014.

Artigo apresentado em 19/08/2018

Aprovado em 15/04/2019

Versão final apresentada em 17/04/2019

Editores chefes: Romeu Gomes, Antônio Augusto Moura da Silva 